Proyecciones Journal of Mathematics Vol. 31, No 1, pp. 25-28, March 2012. Universidad Católica del Norte Antofagasta - Chile

\title{
A simple remark on fields of definition *
}

\author{
RUBÉN HIDALGO \\ UNIVERSIDAD TÉCNICA FEDERICO SANTA MARÍA, CHILE \\ Received : April 2011. Accepted : November 2011
}

\begin{abstract}
Let $K<L$ be an extension of fields, in characteristic zero, with $L$ algebraically closed and let $\bar{K}<L$ be the algebraic closure of $K$ in $L$. Let $X$ and $Y$ be irreducible projective algebraic varieties, $X$ defined over $\bar{K}$ and $Y$ defined over $L$, and let $\pi: X \rightarrow Y$ be a non-constant morphism, defined over $L$. If we assume that $\bar{K} \neq L$, then one may wonder if $Y$ is definable over $\bar{K}$. In the case that $K=\mathbf{Q}, L=\mathbf{C}$ and that $X$ and $Y$ are smooth curves, a positive answer was obtained by González-Diez. In this short note we provide simple conditions to have a positive answer to the above question. We also state a conjecture for a class of varieties of general type.
\end{abstract}

Keywords : Algebraic curves, field of moduli, field of definition.

Subjclass : [2000]14H30, 11G30, 14H25.

*Partially supported by projects Fondecyt 1110001 and UTFSM 12.11.01 


\section{Introduction}

Let us consider a field extension $K<L$, in characteristic zero, where $L$ is algebraically closed, and let $\bar{K}$ be the algebraic closure of $K$ in $L$. Let $X$ be an irreducible projective algebraic variety which is defined over $\bar{K}$ and let $Y$ be an irreducible projective algebraic variety defined over $L$. If $\pi: X \rightarrow Y$ is a non-constant morphism defined over $L$, then we wonder if $Y$ is definable over $\bar{K}$. This is clearly trivial if $K<L$ is a Galois extension and the real problem is when $L \neq \bar{K}$.

In the particular case that $K=\mathbf{Q}, L=\mathbf{C}$, and that $X$ and $Y$ are both non-singular algebraic curves so that $Y$ has genus at least two, it was noticed by González-Diez [2] that the above has a positive answer, that is, the image of Belyi curves are Belyi curves.

The following provides sufficient conditions for the above question to have a positive answer. The arguments of the proof are very simple observations.

Theorem 1. Let $K<L$ be a field extension in characteristic zero, where $L$ is algebraically closed, and let $\bar{K}$ be the algebraic closure of $K$ in $L$. Let $X$ be an irreducible projective algebraic variety defined over $\bar{K}$, let $Y$ be an irreducible projective algebraic variety defined over $L$ and let $\pi: X \rightarrow Y$ be a non-constant morphism, also defined over $L$. If

1. there are, up to birational isomorphisms, only a finite number of varieties with the same algebraic invariants that $Y$ and which are quotients of $X$, and

2. $Y$ can be defined over a finite extension of its field of moduli,

then $Y$ is definable over $\bar{K}$.

Proof. Condition (1) ensures that the field of moduli of $Y$ is $\bar{K}$. In fact, let $\Gamma=\operatorname{Aut}(L / \bar{K})$. If $\sigma \in \Gamma$, then we have the induced non-constant morphism $\pi^{\sigma}: X \rightarrow Y^{\sigma}$. Let $\Gamma_{0}=\left\{\sigma \in \Gamma: Y^{\sigma} \cong Y\right\}$. Assumption (1) asserts that the subgroup $\Gamma_{0}$ has finite index in $\Gamma$. In particular, the fixed field of $\Gamma_{0}$ is a finite extension of $\bar{K}$, so it is equal to $\bar{K}$. If $G=\{\sigma \in$ $\left.\operatorname{Aut}(L / K): Y \cong Y^{\sigma}\right\}$, then the field of moduli of $Y$ is the fixed field of $G$. As $\Gamma_{0}<G$, the field of moduli of $Y$ is contained in the fixed field of $\Gamma_{0}$, which we already noted to be $\bar{K}$. Now assumption (2) asserts that $Y$ is definable over $\bar{K}$. 
The following consequence of Theorem 1 is a mild generalization of the above mentioned Gabino's result in [2] that image of Belyi curves are Belyi.

Corollary 2. Let $K<L$ be a field extension in characteristic zero, where $L$ is algebraically closed, and let $\bar{K}$ be the algebraic closure of $K$ in $L$. Let $X$ be an irreducible projective algebraic curve which is defined over $\bar{K}$, let $Y$ be an irreducible projective algebraic curve, of genus at least 2, defined over $L$ and let $\pi: X \rightarrow Y$ be a non-constant morphism, also defined over $L$. Then $Y$ is definable over $\bar{K}$.

Proof. As $X$ is defined over $\bar{K}$, its singular points are also defined over $\bar{K}$. A blowing-up desingularization of $X$ produces a non-singular model of $X$, also defined over $\bar{K}$. So, from now on, we may assume that $X$ is also non-singular. Similarly, we may assume $Y$ to be non-singular. By a classical theorem of de Franchis [1], there is a finite number of possible quotients $Y^{\sigma}$, up to birational isomorphisms. This asserts assumption (1) of Theorem 1. Assumption (2) of Theorem 1 follows from [3].

A smooth algebraic variety is called of general type if its Kodaira dimension is equal to the dimension of the variety. For instance, a variety with ample canonical bundle is of general type. Also, hypersurfaces in the complex projective $n$-space of degree at least $(n+2)$ are of general type. Abelian varieties and Calabi-Yau manifolds are not of general type as they have Kodaira dimension zero. It is well known that if $Y$ is a smooth algebraic variety of general type, then condition (1) of Theorem 1 holds by a theorem of Tsai [4].

In the introduction of [3] it is claimed that their results may be generalized to classes of varieties for which coarse moduli spaces are known to exists (such as Abelian varieties and surfaces), that is, they can be defined over a finite index extension of their field of moduli. In particular, condition (2) of Theorem 1 will follow from the previous.

In particular, any hypersurface in the complex projective 3-space of degree at least 5 will satisfy both conditions of Theorem 1 . All of the above permits to conjecture the following.

Conjecture 3. Let $K<\mathbf{C}$ and let $\bar{K}$ be its algebraic closure in $\mathbf{C}$. Let $X$ be an irreducible complex projective algebraic variety which is defined over $\bar{K}<\mathbf{C}$ and let $Y$ be an irreducible projective complex algebraic variety of general type defined over $\mathbf{C}$. If $\pi: X \rightarrow Y$ is a non-constant morphism, defined over $\mathbf{C}$, then $Y$ is definable over $\bar{K}$. 


\section{References}

[1] M. de Franchis. Un teorema sulle involutioni irrationali. Rend. Circ. Mat. Palermo 36 (1913), 368.

[2] G. González-Diez. Variations on Belyi's Theorem. Quart. J. Math. 57 (2006), 339-354.

[3] H. Hammer and F Herrlich. A Remark on the Moduli Field of a Curve. Arch. Math. 81 (2003), 5-10.

[4] I. Tsai. Dominating the varieties of general type. J. Reine Angew. Math. 483 (1997), 197-219.

\section{Rubén A. Hidalgo}

Departamento de Matemática,

Universidad Técnica Federico Santa María,

Valparaíso,

Chile

e-mail : ruben.hidalgo@usm.cl 